\title{
INTRA-BEAM SCATTERING AND MINIMUM ACHIEVABLE EMITTANCE IN THE ADVANCED LIGHT SOURCE*
}

\author{
C. Steier, D. Atkinson, J. Byrd, J. Corlett, H. Nishimura, D. Robin, S. de Santis, \\ A. Wolski, Y. Wu, LBNL, Berkeley, CA94720, USA \\ K. Bane, T. Raubenheimer, M. Ross, J. Sheppard, T. Smith, SLAC, Menlo Park, CA94025, USA
}

\begin{abstract}
Intra-beam scattering (IBS) potentially limits the minimum emittance of low-energy storage rings, and this effect strongly influences the choice of energy of damping rings for linear colliders. The Advanced Light Source (ALS) is a third-generation synchrotron light source operating with high-intensity, low-emittance beams at energies up to $2 \mathrm{GeV}$. It can operate with an emittance coupling of below $1 \%$. We present measurements of the beam growth in three dimensions as a function of current, for normalized natural horizontal emittances of approximately $1-10$ $\mathrm{mm}$-mrad at energies of $0.7-1.5 \mathrm{GeV}$, values comparable to the parameters in an NLC damping ring [1]. Using a dedicated diagnostic beamline with an $\mathrm{x}$-ray scintillator imaging system, measurements of the transverse beamsize are made, simultaneously with bunch length measurements using an optical streak camera. The bunch volume growth as a function of bunch current is compared with theoretical estimates, for a parameter space of IBS, where very little experimental data exists so far.
\end{abstract}

\section{ALS MACHINE PARAMETERS}

The ALS is a third generation light source at Lawrence Berkeley National Laboratory, California [2]. The machine with a circumference of $196.8 \mathrm{~m}$ operates typically between 1.5 and $1.9 \mathrm{GeV}$, with $1-1.5 \mathrm{~mA}$ per bunch in approximately 300 bunches in multibunch mode. The ALS lattice is twelve fold symmetric with triple-bend achromat (TBA) unit cells. The typical bunch length in multi bunch operation at $1.5 \mathrm{GeV}$ is $15 \mathrm{ps}$. Up to $35 \mathrm{~mA}$ per bunch are stored in two-bunch mode using the transverse multibunch feedback system to control the single bunch transverse mode coupling instability (TMCI). The TMCI threshold currently is about $12 \mathrm{~mA}$.

In user operation, the bunch volume is intentionally increased both transversely and longitudinally to achieve a reasonable compromise between Touschek lifetime and brightness. Transversely, families of skew quadrupoles are used, to increase the coupling to about $4 \%$. The machine is capable of much smaller coupling ratios, and values below $1 \%$ are routinely achieved.

Longitudinally, harmonic cavities are employed to lengthen the bunch. The RF frequency of the ALS is $500 \mathrm{MHz}$, and the momentum compaction factor for the current user lattice is $1.6 \times 10^{-3}$, but the lattice allows

\footnotetext{
* This work was supported by the U.S. Department of Energy, under Contract No. DE-AC03-76SF00098 and DE-AC03-76SF00515.
}

a fairly flexible control of it. Beam parameters for energies at which IBS studies were performed are shown in table 1 and compared to the design parameters for the NLC damping rings. As one can see, at low energies, the normalized emittances are comparable. The energy spread is always smaller in the ALS than in the NLC DR. The damping times on the other hand are significantly longer at low energies in the ALS. Therefore it is difficult to compare the two cases directly, but comparison of measured IBS growth in the ALS with simulation codes can provide confidence in their predictions for the NLC case.

Table 1: ALS parameters compared to the NLC damping ring design.

\begin{tabular}{|c|c|c|c|c|}
\hline & $\begin{array}{c}\text { Energy } \\
{[\mathrm{GeV}]}\end{array}$ & $\begin{array}{c}\gamma \varepsilon_{x} \\
{[\mathrm{~mm} \mathrm{mrad}]}\end{array}$ & $\begin{array}{c}\frac{\Delta p}{p} \\
{\left[10^{-4}\right]}\end{array}$ & $\begin{array}{c}\tau_{x, E} \\
{[\mathrm{~ms}]}\end{array}$ \\
\hline ALS & 1.9 & 21 & 8 & 8,6 \\
& 1.5 & 10 & 6 & 15,13 \\
& 1.0 & 2.9 & 4 & 52,44 \\
& 0.7 & 1.0 & 2.8 & 147,128 \\
\hline NLC DR & 1.98 & 2.3 & 9.1 & $5,2.5$ \\
\hline
\end{tabular}

\subsection{Machine set-up}

Overall, the lattice of the ALS is understood and characterized very well (linear, nonlinear and coupling). There is a simple procedure for the machine setup and the results are very reproducible.

To evaluate the symmetry of the lattice and indirectly measure the optical functions around the ring, orbit response matrix measurements are carried out regularly. The data is then analyzed using the code LOCO [3, 4]. Using the results, the residual beta-beating can be corrected to less than $1 \% \mathrm{rms}$, and is typically below $3 \%$. The typical residual vertical dispersion is about $5 \mathrm{~mm}$ rms. Emittance measurements made with an x-ray beamline (see section 2.1) agree to better than $5 \%$ with calculated natural emittances.

\section{DIAGNOSTICS}

\subsection{Diagnostic beamline 3.1}

There is a dedicated diagnostics beamline at the ALS (designated 3.1) with an imaging x-ray optics. The source point is in the first dipole magnet of a TBA cell. At this point $\beta_{y}$ is very large, while $\beta_{x}$ and the dispersion are relatively small $\left(\beta_{x}=0.4 \mathrm{~m}, \eta_{x}=0.03 \mathrm{~m}, \beta_{y}=19 \mathrm{~m}\right)$. The spurious vertical dispersion is fairly constant over time $\left(\eta_{y}=-0.01 \mathrm{~m}\right)$. 
The imaging optics are shown in figure 1. The optical system produces a 1:1 image of the source on a bismuth germinate crystal. At $1.5 \mathrm{GeV}$, the optical resolution of the system as determined by lifetime and beamsize measurements is less than $10 \mu \mathrm{m}$. Therefore, the resolution correction is insignificant for the smallest beamsizes measured in the IBS studies. Due to the dramatic drop in the critical energy of the photons emitted from the bending magnet, the resolution at lower energies seems to change and requires further study. Carbon neutral density filters are used to avoid saturation of the optics and to block visible light. An optical microscope magnifies the visible light image from the crystal scintillator onto a CCD camera, with a pixel resolution of approximately $4 \mu \mathrm{m}$.

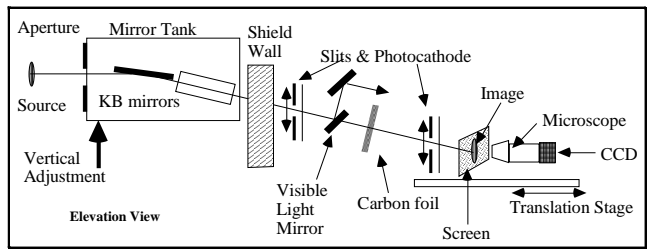

Figure 1: Diagnostic beamline 3.1.

The output of the CCD camera is digitized and a 2-D Gaussian fit is used to analyze the transverse beam distribution. Since the betatron axes are not necessarily identical with the horizontal and vertical planes at the source point, the fitting routine must find these axes before deriving $\sigma_{x}$ and $\sigma_{y}$. This effect can be significant, depending on coupling. The error in the fit is typically $\pm 0.5 \mu \mathrm{m}$.

Inserting a mirror into the optical path allows visible light to be imaged into a Hamamatsu streak camera. The streak camera output is recorded and analyzed offline.

\section{MEASUREMENTS}

Measurements of transverse and longitudinal beam sizes are made in single-bunch mode. A single-bunch current of $1 \mathrm{~mA}$ in the ALS corresponds to $0.67 \mathrm{nC}$ or $4.2 \times 10^{9}$ electrons. Beam current and beam dimensions are recorded as the current decays naturally (for higher current), and by knockout using a pulsed magnet when the lifetime becomes large. The emittance coupling is first minimized by careful machine set-up and use of the four skew-quardupole families in the ALS. To control the emittance coupling during the measurements both the tunes and the width of the coupling resonance are changed.

\section{$3.1 \quad 1.5 \mathrm{GeV}$}

Since the lattice is very well characterized between 1.5 and $1.9 \mathrm{GeV}$, where the ALS is normally operated for users, and the resolution of the diagnostic elements for those energies was known, the first IBS measurements were carried out at $1.5 \mathrm{GeV}$. On the other hand it was already known, that potential well distortion and microwave instability are fairly strong at $1.5 \mathrm{GeV}$ [5]. Figure 2 shows the bunch length as a function of current for different coupling factors and RF voltages. The bunch length for small and large coupling is identical whereas growth of the bunch length due to IBS should be a strong function of the transverse beamsizes. Therefore, one can conclude that IBS is negligible.

Simultaneously an increase of transverse bunch sizes with current is observed. All measurements can be consistently explained by a combination of potential well distortion and energy spread increase due to microwave instability and an impedance model was derived based on these and earlier measurements [5].

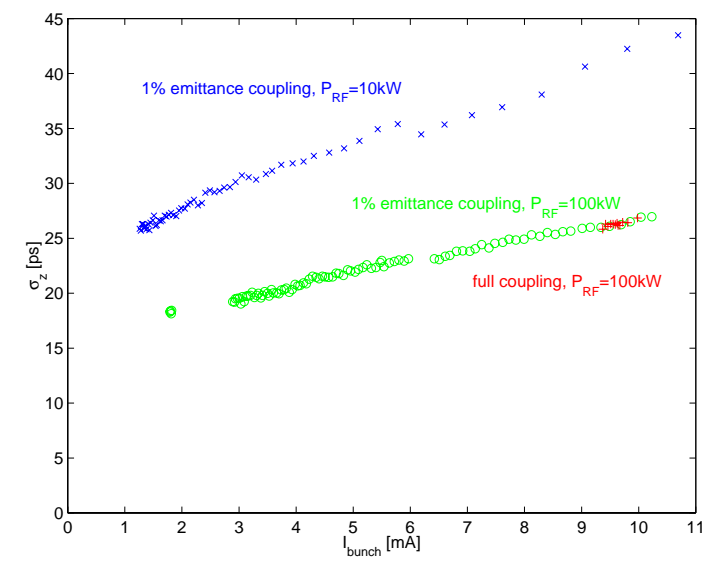

Figure 2: Bunch length measurements at $1.5 \mathrm{GeV}$.

IBS calculations using both a modified BjorkenMtingwa and the Piwinski formalism [6, 7] have been made for the ALS lattice using (in the $1.5 \mathrm{GeV}$ case) the impedance model derived from the measurements to take potential well distortion and energy spread increase from microwave instability into account. The simulations predict an increase due to IBS just below our measurement errors. The measurements therefore provide an upper boundary and the bunch volume growth due to IBS in this case cannot be significantly larger than the simulations predict.

\section{$3.21 .0 \mathrm{GeV}$}

Since the IBS effects scale significantly faster with energy than impedance driven effects, one would expect that they are more visible at lower energies. Figure 3 shows the measured horizontal, vertical and longitudinal bunch size at $1.0 \mathrm{GeV}$ as a function of bunch current for two different conditions of emittance coupling. The bunch length is significantly (15-45\%) increased in the low-coupling case, which is a clear indication for intra-beam scattering.

The beamsizes at the lowest bunch current of about $0.25 \mathrm{~mA}$, correspond to normalized emittances of $\gamma \varepsilon_{x}=$ $4 \times 10^{-6} \mathrm{~m}$-rad and $\gamma \varepsilon_{y}=0.07 \times 10^{-6} \mathrm{~m}$-rad, assuming natural energy spread (since the energy spread is increased as well, this overestimates the emittances), which is fairly close to the NLC DR design parameters. For the high-coupling case, the change in vertical beamsize as a function of current is caused by the tune shift with bunch current due to the broadband transverse impedance, moving the tune off the coupling resonance.

To compare the measurement results with simulations is more difficult than at $1.5 \mathrm{GeV}$. The reason is that now 

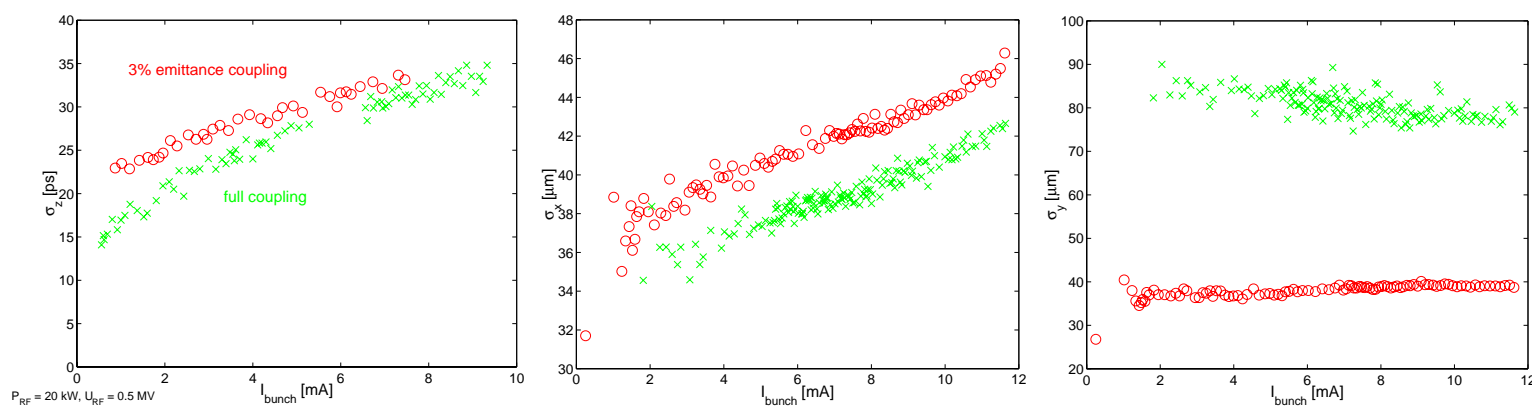

Figure 3: Bunch length and transverse beam size measurements at $1.0 \mathrm{GeV}$.

impedance driven effects are not well characterized. Therefore we compared the growth in overall bunch volume as measured and as predicted by IBS simulations without taking microwave instability or potential well distortion into account. Even though this does not allow a precise test of the simulations, it still gives a good check whether the predictions are about correct.

Assuming no contribution from microwave instability or potential well distortion, the simulations predict an increase of the transverse emittances and the energy spread by a factor of 3 to 5 over the range of $0-10 \mathrm{~mA}$ per bunch, resulting in a bunch volume increase by a factor of 5 to 11 . We observe an increase of approximately a factor of 6 .

\section{$3.3 \quad 0.7 \mathrm{GeV}$}

Continuing to lower energies, where the IBS effects should become more and more dominant, the same set of measurements as at $1.0 \mathrm{GeV}$ was performed at $0.7 \mathrm{GeV}$. Due to limitations in the resolution of the x-ray optics resulting from the dramatic drop in the critical energy emitted from the bend magnet at such low energies, the measurement of transverse beamsizes is not reliable. A more detailed study has to be performed, to quantify the resolution limit at this energy and to determine, whether the measurement data can be corrected for the resolution.

The bunch length was again measured for different RF voltages, as a function of beam current, on and off the coupling resonance. Figure 4 shows the variation of the bunch length under these conditions. The increase in bunch length in the off-resonance case is indeed larger than at $1.0 \mathrm{GeV}$ and is again a clear evidence of IBS.

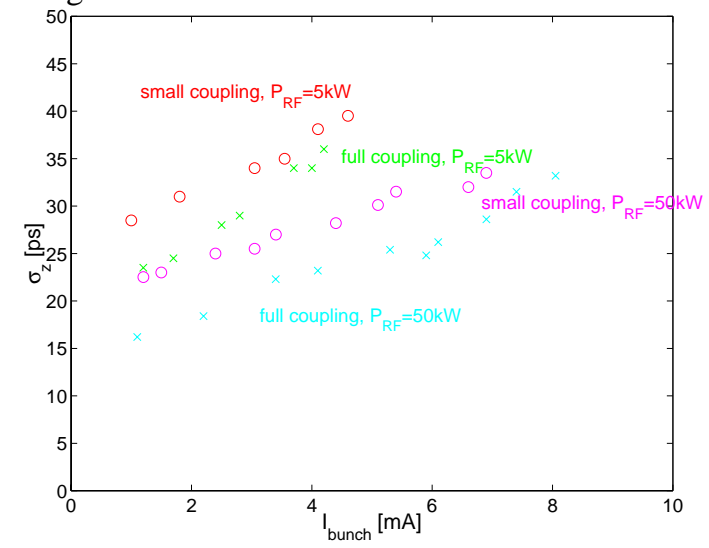

Figure 4: Bunch length measurements at $0.7 \mathrm{GeV}$.

\section{SUMMARY/OUTLOOK}

The desire to achieve low emittances at a relatively low energy in the NLC damping rings to reduce its costs has led to IBS studies at machines with similar parameters. While especially the damping times at the ALS do not match those of the NLC damping rings, the emittance is similar and a comparison with theory will allow a better understanding and confidence in IBS calculations. In addition, differences in the impedance between machines complicate comparisons. Emittance growth has also been studied at the Accelerator Test Facility at KEK, Japan [8]. More detailed calculations of IBS are currently in progress. Measurements to date are consistent with preliminary calculations.

For future measurements, we plan to use an additional diagnostic beamline, with different optical functions at the source point. First tests were carried out and a cross calibration with the existing diagnostic beamline was done. Further studies of the resolution limits of the new beamline are needed. Data from multiple observation points should allow the separation of energy spread and transverse emittance making the comparison with simulations easier. It is planned to continue the measurements and to compare them with improved simulations.

\section{REFERENCES}

[1] 'Zeroth-Order Design Report for the Next Linear Collider', The NCL Design Group, SLAC Report 474, May 1996.

[2] 1-2 GeV Synchrotron Radiation Source Conceptual Design Report, LBNL publication PUB-5172 Rev. (1986)

[3] J. Safranek, Nucl. Instr. and Meth. A388, 27 (1997)

[4] C. Steier, D. Robin, 'Fully coupled analysis of orbit response matrices at the ALS', EPAC 2000, Vienna.

[5] J. Byrd, 'Observations of single bunch collective effects in the ALS', Beam Instability Workshop, ESRF, March 2000.

[6] J.D. Bjorken and S.K. Mtingwa, 'Intrabeam Scattering', Part. Accel. 13 (1983), 115

[7] A. Piwinski, 'Intrabeam Scattering', Proc. HEACC 1974

[8] J. Urakawa, 'Experimental Results and Technical Research and Development at ATF (KEK)', Proc. EPAC 2000, Vienna, 2000. 